\title{
THE IMPACT ON SCALING ON THE PAIR-WISE COMPARISON OF THE ANALYTIC HIERARCHY PROCESS
}

\author{
Yuji Sato \\ Department of Policy Science, Matsusaka University \\ 1846, Kubo, Matsusaka, Mie, 515-8511 - Japan \\ ysatoh@matsusaka-u.ac.jp
}

Keywords: linear scale, power scale, consistency index, rank reversal, discriminating sensitivity

Summary: The objective of this study is to find the scale of the Analytic Hierarchy Process (AHP) appropriate for representing decision maker's perception. Specifically, two scales, linear scale and power scale, employed in the pair-wise comparison of the AHP are evaluated. The results offer some evidence that the power scale is preferable to the linear scale as the judgment scale.

\section{Introduction}

One of the most popular methods for decision making has been the Analytic Hierarchy Process (AHP) developed by Thomas L. Saaty (Saaty, 1977). The advantage to the AHP is that data on decision-makers' (DMs) pair-wise comparisons are aggregated, and the degree of importance of each alternative is quantified. This quantification results in not only the identification of the most important alternative but also the ranking of all alternatives for each DM.

Two issues surround the use of the AHP. The first issue occurs because the allowable upper bound of consistency index (CI) is $10 \%$ of the RI. Pair-wise comparison matrices with CIs greater than this critical value are therefore not accepted due to their inconsistency. Consequently, in case applying the AHP to questionnaire on public opinion, the more samples whose CI exceed the limit, the fewer the samples available for analysis (henceforth, CI problem). The second issue surrounding the AHP involves the range of $a_{i j}$, the relative weight of alternative $i$ ( $i$ for short) to $j$. When the range of $a_{i j}$ is expanded (reduced) from 1-9 to 1-15 (1-5), the Frobenius root and its corresponding eigenvector changes, resulting in the alternation of the direction of the eigenvector and the rank of the priority of alternatives (Schenkerman, 1994) (henceforth, rank-reversal problem).

In addition to the above two traditional issues, one additional problem must be noted. If the range of $a_{i j}$ were to be too reduced, then CI would converge to 0, making it impossible for the AHP to discriminate an important alternative from others. Since discriminating the most important alternative is one of the principal purposes of the AHP, verifying how sensitively the AHP discriminates an important alternative from others when $a_{i j}$ s are scattered is essential (henceforth, discriminating-sensitivity problem).

In the AHP, a pair-wise comparison and a scale used in its procedure play a key role quantifying each DM's feeling; therefore, which scale should be used in the process of a pair-wise comparison is the most controversial issue concerning the refinement of this method. Indeed, the three aforementioned problems (the CI problem, the rank-reversal problem and the discriminating-sensitivity problem) are deeply related to the scale. The 1-9 linear scale, advocated by Saaty (Saaty, 1980) long considered the standard of the AHP, has been widely criticized in the literature, primarily because of the CI problem and the rank-reversal problem.

To overcome the deficiencies of Saaty's scale, various judgment scales for a pair-wise comparison have been proposed and evaluated to date. Aupetit and Genest proposed reducing the range of the linear scale to 1-5 (Aupetit and Genest, 1993); Harker and Vargas proposed extending the range of the linear scale to 1-13 and 1-50 (Harker and Vargas, 1987). In addition to these linear scales, Harker and Vargas also 
proposed two non-linear scales (quadratic and irrational) (Harker and Vargas, 1987). Lootsma claimed the superiority of the power scale to the 1-9 linear scale (Lootsma, 1989 and 1991). Saaty's and Lootsma's scales have often been compared and have generated much discussion.

Saaty verified the effectiveness of his 1-9 scale through many physical experiments, each of which had a theoretical value (Saaty, 1983). The AHP, however, deals with decision making on subjective issues, making it difficult to determine theoretically whether or not each DM's preference for alternatives derived from the AHP accurately represents each DM's feeling. Taking into account the fact that the AHP deals with not only objective issues that can be quantified but also subjective issues that do not have theoretical values, the effectiveness of a scale must be verified empirically through actual applications to subjective issues. In this study, we focus on comparing the effectiveness of Saaty's 1-9 linear scale and the power scale from the perspective of appropriateness for representing each DM's perception, where the criteria of appropriateness are consistency, robustness with respect to the change of the range of a scale, and discriminating sensitivity.

In this study, two types of data are used to evaluate the two scales. One is randomly generated data, five sizes of matrices (from $3 \times 3$ to $7 \times 7$ ) whose elements are generated in accordance with uniform randomness (random sample); the other is actual data, a matrix $(4 \times 4)$ whose elements are obtained from a 1997 survey of public opinion on the Japanese election results of October 1996 (actual sample). This election represented the first time the election law of the House of Representatives switched from a multi-member electorate system to a single-member electorate system. The survey was carried out four months following the election. The data obtained from this survey might thus be considered an accurate reflection of each respondent's subjective judgment and serve as an ideal actual sample for evaluating scales used in a pair-wise comparison.

\section{Set-up for the empirical test}

\subsection{Definition of the linear ant the power scales for a pair-wise comparison}

A scale employed in a pair-wise comparison can be defined as a function mapping the result of a pair-wise comparison between two alternatives to reciprocal value. Let $\boldsymbol{I}$ be a linearly symmetric interval, whose center (set as 0 ) represents two alternatives of equal importance with the magnitude of importance symmetrically distributed on both sides (set, + and - ). Further let $x_{i j} \in\{-k, \ldots,-1,0,1, \ldots$, $k\} \subset I\left(x_{i j}+x_{j i}=0\right.$ and $\left.i, j=1, \ldots, n\right)$ be pair-wise comparison data representing each result of pair-wise comparisons between an alternative $i$ ( $i$, for short) and $j$. If we describe the relative weight of $\boldsymbol{i}$ to $\boldsymbol{j}$ as $\boldsymbol{a}_{\boldsymbol{i}}$, then a scale can be defined as the monotone, increasing function $f: x_{i j} \rightarrow a_{i j}$, which satisfies $f\left(x_{i j}\right) \cdot f\left(x_{j i}\right)=1$.

\section{Definition 1: Linear Scale}

$$
f_{L}\left(x_{i j} ; c\right)=\left\{\begin{array}{ll}
c x_{i j}+1 & x_{i j} \geq 0 \\
1 /\left(-c x_{i j}+1\right) & x_{i j}<0
\end{array} \quad\left(\text { for } \forall_{c>0}\right)\right.
$$

\section{Definition 2: Power Scale}

$$
f_{P}\left(x_{i j} ; m\right)=m^{x_{i j}} \quad(\text { for } \forall m>1)
$$

Henceforth, we describe the pair-wise comparison matrix generated by the linear scale, $f_{L}\left(x_{i j} ; c\right)$, as $A_{L}$ $=\left(f_{L}\left(x_{i j} ; c\right)\right)$ and that generated by the power scale, $f_{P}\left(x_{i j} ; m\right)$, as $A_{P}=\left(f_{P}\left(x_{i j} ; m\right)\right)^{1}$. Saaty's linear scale corresponds to the case $k=8$ for $x_{i j}$ and $c=1$ in Equation (1); Aupetit-Genest's linear scale corresponds to that of $k=12$ and $c=1$; and Harker-Vargas's linear scale corresponds to that of $k=8$ and $c=0.5$. However, in the AHP, since Saaty's 1-9 linear scale has been employed as a standard, we fix $k=8$ and $c=1$ for purposes of this study.

1 Lootsma employed the similar definition of the power scale; the pair-wise comparison data $x_{i j}$ was set as $x_{i j} \in\{2 k \mid k=0, \ldots, 4\}$ (Lootsma, 1991). 
In the definition of the power scale, the size of $m$ greatly affects the results. If $m \rightarrow 1$, then a weight vector converges to $1 / n \cdot 1^{2}$, resulting in no useful information concerning the degree of importance of alternatives. In contrast, if $m$ is set large, then the values of pair-wise comparisons diverge extremely or converge to 0 , resulting in a deviation from individuals' feelings. One reasonable way of setting $m$ is to set the maximum of $f_{P}\left(x_{i j} ; m\right)$ the same as that of $f_{L}\left(x_{i j} ; 1\right)$, which results in $m^{8}=9(m \cong 1.3161)$; however, under this condition, $f_{P}\left(x_{i j} ; 1.3161\right)<f_{L}\left(x_{i j} ; 1\right)$ when $0<x_{i j}<8$ because $f_{P}$ is a convex function. In this case, CI of $A_{P}=\left(f_{P}\left(x_{i j} ; m\right)\right)$ tends to be smaller than that of $A_{L}=\left(f_{L}\left(x_{i j} ; c\right)\right)$, making the comparison about the size of CI unfair. Therefore, in this study, we fix $\boldsymbol{m}(\boldsymbol{c})$, so as to satisfy the following equation:

$$
\int_{0}^{8}(c x+1) d x=\int_{0}^{8} m^{x} d x .
$$

From Equation (3), the approximate value of $m(1)$ is calculated as $m(1)=1.3945$. Henceforth, the constant $c$ and $m(c)$ are represented by 1 and $m(1)$ respectively, and the functions $f_{L}\left(x_{i j} ; c\right)$ and $f_{P}\left(x_{i j} ; m\right)$ are described as $f_{L}$ and $f_{P}$, for short. Insofar as the calculation of weight is concerned, we follow the eigenvalue method, because the weight calculated by the log-least-square method and that calculated by the geometric-mean method coincide (Crawford and Williams, 1985), and that the latter coincides the weight calculated by the eigenvalue method.

\subsection{Samples}

In this study, we use two qualitatively different types of data to evaluate the appropriateness of the two above-defined scales for representing each DM's feeling. One type of data is randomly generated, five sizes of matrices (from $3 \times 3$ to $7 \times 7$ ) whose elements are generated in accordance with uniform randomness (random sample); the other is actual data, a matrix $(4 \times 4)$ whose elements are obtained from a 1997 survey of public opinion on the Japanese election results of October 1996 (actual sample).

\subsubsection{Random sample}

The random sample is generated in the following way. First, we select 20000 sets $(5000$ sets for $3 \times 3$ matrix) of random pair-wise comparison data $\left\{x_{i j}\right\}_{r}(i, j=1, \ldots, h$ and $h=3, \ldots, 7)$ from $\{-8, \ldots,-1,0,1, \ldots$, $8\}$ in accordance with uniform randomness, and next map $\left\{x_{i j}\right\}_{r}$ by $f_{L}$ and $f_{P}$, generating five types (from 3 $\times 3$ to $7 \times 7$ ) of reciprocal pair-wise comparison matrices $A_{L}$ and $A_{P}$, respectively. In this study, these two sets of five types of 20000 matrices are defined as the random sample and used in the evaluation of $f_{L}$ and $f_{P}$.

\subsubsection{Actual sample}

The actual sample is obtained from a 1997 survey of public opinion on the Japanese election results of October 1996. The total number of responses is 796 and the response ratio is $83.1 \%$. In this survey, the following three questions are formatted in the AHP system:

Q1: the criteria used in the choice of the candidate in the election

Q2: the reason why respondents were non-partisan

Q3: the criteria used in the choice of the candidate in the previous election in July 1993

Each question asks respondents about subjective issues that could not be measured theoretically. In these questions, respondents are asked to conduct a pair-wise comparison of each criterion in accordance with the segmental method, where $I=\{-8, \ldots,-1,0,1, \ldots, 8\}$.

The actual pair-wise comparison data $\left\{\boldsymbol{x}_{i j}\right\}_{d}\left(i, j=1, \ldots, 4, x_{i j} \in I\right)$ is mapped by $f_{L}$ and $f_{P}$, generating a 4 $\times 4$ reciprocal pair-wise comparison matrix $A_{L}$ and $A_{P}$, respectively. $A_{L}$ and $A_{P}$ are defined as the actual sample and used in the evaluation of $f_{L}$ and $f_{P}$ in this study. The size of the actual sample is 1409 , which is the total number of qualified answers ${ }^{3}$ obtained from the above three questions.

\footnotetext{
2 a vector whose elements are all 1

3 the CIs of the answers are smaller than 0.15
} 


\subsection{Testing item}

In this research, using the two types of samples defined in Section 2.2, we evaluated $f_{L}$ and $f_{P}$ from the following perspectives: distribution of CI, rank-reversal problem, and discriminating-sensitivity problem.

\section{Comparison between the linear and the power scales using the random sample}

\subsection{CI}

Let $\boldsymbol{r} \mathbf{C I}_{\boldsymbol{L}(\boldsymbol{h})}$ and $\boldsymbol{r} \mathbf{C I} \mathbf{I}_{P(h)}$ denote the $\mathbf{C I}$ obtained from the random sample, $A_{L}$ and $A_{P}$, whose matrix sizes are $h \times h$, respectively. Furthermore, let $\mathbf{M}\left(r \mathbf{C I} \mathbf{I}_{L(h)}\right)$ and $\mathbf{M}\left(r \mathbf{C I} \mathbf{I}_{P(h)}\right)$ denote the mean of $r \mathbf{C I} \mathbf{I}_{L(h)}$ and $r \mathbf{C I} \mathbf{I}_{P(h)}$, respectively, over the entire random sample. Table 1 summarizes $m(c), \mathrm{M}\left(r \mathrm{CI}_{L(h)}\right)$ and $\mathrm{M}\left(r \mathrm{CI}_{P(h)}\right)$ corresponding to each $c=0.5,1,1.5,2,3,4$ and 5 in accordance with Equation (3). As can be seen, each pair of $\mathrm{M}\left(r \mathrm{CI}_{L(h)}\right)$ and $\mathrm{M}\left(r \mathrm{CI}_{P(h)}\right)$ was nearly equal. However, $r \mathrm{CI}_{L(h)}$ and $r \mathrm{CI}_{P(h)}$ had different distributions for each random-sample size $h$. Therefore, $f_{L}$ and $f_{P}$ could not be considered equivalent scales just because $\mathrm{M}\left(r \mathrm{CI}_{L(h)}\right)$ nearly equaled $\mathrm{M}\left(r \mathrm{CI}_{P(h)}\right)$. Thus, we focused on the top $1 \%, 3 \%, 5 \%, 7 \%, 10 \%, 15 \%, 20 \%$ and $25 \%$ values of $r \mathrm{CI}_{L(h)}$ and $r \mathrm{CI}_{P(h)}$, and defined the threshold point (T-point, for short) as the border value of each $r \mathrm{CI}_{L(h)}$ and $r \mathrm{CI}_{P(h)}$. As Table 2 shows, $r \mathrm{CI}_{L(h)}>r \mathrm{CI}_{P(h)}$ held at almost all the T-points for each sample size $h$, implying that the CI of a pair-wise comparison matrix generated by $f_{L}$ was greater than that generated by $f_{P}$ for each T-point. In this study, $f_{L}$ and $f_{P}$ were evaluated based on this T-point.

\begin{tabular}{c|ccccccc}
$\mathbf{M}\left(\boldsymbol{r} \mathbf{C I}_{\boldsymbol{L}(\boldsymbol{h})}\right)$ and $\mathbf{M}\left(\boldsymbol{r} \mathbf{C I}_{\boldsymbol{P}(\boldsymbol{h})}\right)$ & \multicolumn{1}{c}{ Table 1 } \\
\hline$c$ & 0.5 & 1 & 1.5 & 2 & 3 & 5 \\
$m(c)$ & 1.2687 & 1.3945 & 1.4792 & 1.5438 & 1.6406 & 1.7132 \\
\hline $\mathrm{M}\left(r \mathrm{CI}_{L(3)}\right)$ & 0.2378 & 0.5270 & 0.8106 & 1.088 & 1.627 & 2.153 \\
$\mathrm{M}\left(r \mathrm{CI}_{P(3)}\right)$ & 0.2511 & 0.5382 & 0.804 & 1.052 & 1.513 & 1.938 & 2.7717 \\
\hline $\mathrm{M}\left(r \mathrm{CI}_{L(4)}\right)$ & 0.3925 & 0.8996 & 1.400 & 1.916 & 2.945 & 3.968 & 4.984 \\
$\mathrm{M}\left(r \mathrm{CI}_{P(4)}\right)$ & 0.4028 & 0.9013 & 1.400 & 1.880 & 2.788 & 3.652 & 4.484 \\
\hline $\mathrm{M}\left(r \mathrm{CI}_{L(5)}\right)$ & 0.4711 & 1.111 & 1.776 & 2.451 & 3.825 & 5.193 & 6.563 \\
$\mathrm{M}\left(r \mathrm{CI}_{P(5)}\right)$ & 0.5051 & 1.168 & 1.832 & 2.487 & 3.767 & 5.008 \\
\hline $\mathrm{M}\left(r \mathrm{CI}_{L(6)}\right)$ & 0.5301 & 1.257 & 2.019 & 2.793 & 4.358 & 5.933 \\
$\mathrm{M}\left(r \mathrm{CI}_{P(6)}\right)$ & 0.5719 & 1.340 & 2.125 & 2.909 & 4.461 & 5.991 \\
\hline $\mathrm{M}\left(r \mathrm{CI}_{L(7)}\right)$ & 0.6160 & 1.457 & 2.336 & 3.229 & 5.033 & 6.847 & 7.501 \\
$\mathrm{M}\left(r \mathrm{CI}_{P(7)}\right)$ & 0.6762 & 1.620 & 2.618 & 3.640 & 5.725 & 7.843 \\
\hline
\end{tabular}

\begin{tabular}{|c|c|c|c|c|c|c|c|c|c|c|}
\hline \multicolumn{9}{|c|}{$r C I_{L(h)}$ and $r C I_{P(h)}$ corresponding to each T-point $(c=1, m=m(1))$} & \multicolumn{2}{|r|}{ Table 2} \\
\hline Matrix Size & & & & & & & & & & \\
\hline T-point & $r \mathrm{CI}_{L(3)}$ & $r \mathrm{CI}_{P(3)}$ & $r \mathrm{CI}_{L(4)}$ & $r \mathrm{CI}_{P(4)}$ & $r \mathrm{CI}_{L(5)}$ & $r \mathrm{CI}_{P(5)}$ & $r \mathrm{CI}_{L(6)}$ & $r \mathrm{CI}_{P(6)}$ & $r \mathrm{CI}_{L(7)}$ & $r \mathrm{CI}_{P(7)}$ \\
\hline $1 \%$ & 0 & 0 & 0.04741 & 0.03277 & 0.1752 & 0.1482 & 0.3486 & 0.3168 & 0.4834 & 0.4366 \\
\hline $3 \%$ & 0.000991 & 0 & 0.08662 & 0.06862 & 0.2446 & 0.2319 & 0.4819 & 0.4507 & 0.6574 & 0.5839 \\
\hline $5 \%$ & 0.003511 & 0.006150 & 0.1141 & 0.09807 & 0.3002 & 0.2936 & 0.5628 & 0.5169 & 0.7536 & 0.6709 \\
\hline $7 \%$ & 0.006296 & 0.006150 & 0.1448 & 0.1251 & 0.3472 & 0.3412 & 0.6293 & 0.5800 & 0.8285 & 0.7534 \\
\hline $10 \%$ & 0.01230 & 0.006150 & 0.1781 & 0.1648 & 0.4129 & 0.4077 & 0.7097 & 0.6494 & 0.9169 & 0.8377 \\
\hline $15 \%$ & 0.02681 & 0.02468 & 0.2318 & 0.2234 & 0.5178 & 0.4990 & 0.8123 & 0.7518 & 1.019 & 0.9601 \\
\hline $20 \%$ & 0.04701 & 0.02468 & 0.2865 & 0.2899 & 0.6186 & 0.5796 & 0.8991 & 0.8350 & 1.099 & 1.062 \\
\hline $25 \%$ & 0.06781 & 0.05581 & 0.3421 & 0.3458 & 0.7136 & 0.6610 & 0.9773 & 0.9088 & 1.171 & 1.159 \\
\hline
\end{tabular}

\subsection{Rank-reversal problem}

With respect to the change of $c$ and $m(c)$ defining the function $f$, the range of $a_{i j}$ changes, resulting in the perturbation of the Frobenius root, and the concomitant alternation of the direction of the corresponding eigenvector. This alternation, in turn, reverses the preference order of alternatives, resulting in the rank-reversal problem. If a rank reversal occurs between primal alternatives, derived preference order cannot be trustworthy.

In the analysis of the rank-reversal problem, we randomly extracted 1000 samples from each set of 20000 random samples $A_{L}$ and $A_{P}$ from $3 \times 3$ to $7 \times 7$ defined in Section 2.2.1, and evaluated the robustness of $f_{L}$ 
and $f_{P}$ with respect to the change of $c$ and $m(c)$ as the following range:

$$
c: 0 \leq c \leq 10, \quad m(c): 1 \leq m(c) \leq 3 .
$$

The ranges of $c$ and $m(c)$ in Equation (4), corresponding to the wider range of $a_{i j}$ mapped by $f_{L}$ and $f_{P}$ than that mapped by the previously proposed scales $f$ are wide enough for evaluating $f_{L}$ and $f_{P}$. In the comparison of $f_{L}$ and $f_{P}$, all samples are categorized by each $1 \%, 3 \%, 5 \%, 7 \%, 10 \%, 15 \%, 20 \%$ and $25 \%$-T-point defined in Section 3.1, and in each category, $f_{L}$ and $f_{P}$ are evaluated as to whether or not rank reversal occurs. Among the above-mentioned 1000 samples, $\mathbf{A}$ and $\mathbf{A}^{\prime}$ denote the cumulative number of samples generated by $f_{L}$ and $f_{P}$ to each T-point, respectively. Furthermore, among the A and A' samples in each category, B and B' respectively denote the cumulative number of samples where the rank reversal occurs ${ }^{4}$ with respect to the change of $\boldsymbol{c}$ and $\boldsymbol{m}(\boldsymbol{c})$ over the range shown in Equation (4). Table 3 shows (A, B), (A', B') for $h=4$. As for the total number of rank-reversal samples, B $\geq \mathrm{B}^{\prime}$ for $h=3,4$ and $\mathrm{B} \leq \mathrm{B}$ ' for $h=5, \ldots, 7$; however, for samples with small CIs, B $\geq \mathrm{B}$ ' held in every matrix size $h=3, \ldots, 7$, implying that $f_{P}$ was more robust than $f_{L}$ for the change of $c$ and $m(c)$. Figures 1.1 and 1.2 show rank-reversal examples whose CIs are small and the rank reversal occurs in the neighborhood of $c=1$.

Number of samples and their ratios: Rnak reversed

\begin{tabular}{|c|c|c|c|c|c|c|c|c|c|c|c|c|c|c|c|c|c|c|c|c|}
\hline \multirow{3}{*}{$\frac{\frac{\text { Matrix Size }}{\text { Scale }}}{\text { T-point }}$} & \multicolumn{4}{|c|}{$3 \times 3$} & \multicolumn{4}{|c|}{$4 \times 4$} & \multicolumn{4}{|c|}{$5 \times 5$} & \multicolumn{4}{|c|}{$6 \times 6$} & \multicolumn{4}{|c|}{$7 \times 7$} \\
\hline & \multicolumn{2}{|c|}{$f_{L}$} & \multicolumn{2}{|c|}{$f_{P}$} & \multicolumn{2}{|c|}{$f_{L}$} & \multicolumn{2}{|c|}{$f_{P}$} & \multicolumn{2}{|c|}{$f_{L}$} & \multicolumn{2}{|c|}{$f_{P}$} & \multicolumn{2}{|c|}{$f_{L}$} & \multicolumn{2}{|c|}{$f_{P}$} & \multicolumn{2}{|c|}{$f_{L}$} & \multicolumn{2}{|c|}{$f_{P}$} \\
\hline & $\mathrm{A}$ & $\mathrm{B}$ & $\overline{A^{\prime}}$ & $\overline{\mathrm{B}^{\prime}}$ & $\mathrm{A}$ & B & $\overline{A^{\prime}}$ & $\mathrm{B}^{\prime}$ & $\mathrm{A}$ & B & $\overline{\mathrm{A}^{\prime}}$ & $\mathrm{B}^{\prime}$ & $\mathrm{A}$ & B & $\overline{\mathrm{A}^{\prime}}$ & $\mathrm{B}^{\prime}$ & $\mathrm{A}$ & B & $\overline{\mathrm{A}^{\prime}}$ & $\mathrm{B}^{\prime}$ \\
\hline $1 \%$ & 21 & 0 & 47 & 0 & 13 & 0 & 16 & 0 & 13 & 0 & 8 & 0 & 10 & 2 & 7 & 0 & 6 & 0 & 2 & 0 \\
\hline $3 \%$ & 41 & 0 & 47 & 0 & 36 & 4 & 37 & 0 & 23 & 0 & 30 & 0 & 23 & 3 & 22 & 0 & 20 & 4 & 13 & 0 \\
\hline $5 \%$ & 59 & 0 & 144 & 0 & 50 & 8 & 63 & 0 & 51 & 0 & 55 & 0 & 38 & 4 & 36 & 1 & 41 & 7 & 27 & 0 \\
\hline $7 \%$ & 75 & 0 & 144 & 0 & 84 & 15 & 79 & 0 & 72 & 5 & 77 & 1 & 58 & 6 & 60 & 2 & 60 & 11 & 58 & 0 \\
\hline $10 \%$ & 106 & 0 & 144 & 0 & 113 & 20 & 116 & 0 & 115 & 16 & 118 & 5 & 89 & 6 & 86 & 5 & 103 & 23 & 99 & 0 \\
\hline $15 \%$ & 169 & 0 & 231 & 0 & 169 & 26 & 154 & 0 & 180 & 22 & 173 & 8 & 125 & 12 & 123 & 11 & 159 & 30 & 156 & 13 \\
\hline $20 \%$ & 217 & 0 & 231 & 0 & 208 & 34 & 210 & 3 & 230 & 35 & 230 & 16 & 166 & 20 & 177 & 15 & 225 & 40 & 223 & 35 \\
\hline $25 \%$ & 275 & 7 & 336 & 0 & 260 & 42 & 265 & 3 & 272 & 44 & 278 & 35 & 228 & 33 & 220 & 16 & 288 & 46 & 308 & 45 \\
\hline $100 \%$ & 1000 & 36 & 1000 & 2 & 1000 & 291 & 1000 & 285 & 1000 & 127 & 1000 & 196 & 1000 & 161 & 1000 & 274 & 1000 & 201 & 1000 & 368 \\
\hline
\end{tabular}

Example 1

$A_{L}=\left(\begin{array}{cccc}1 & 4 & 2 & 1 \\ 1 / 4 & 1 & 1 / 8 & 1 / 9 \\ 1 / 2 & 8 & 1 & 1 \\ 1 & 9 & 1 & 1\end{array}\right), r \mathrm{CI}_{L(4)}=0.0659, c=1$

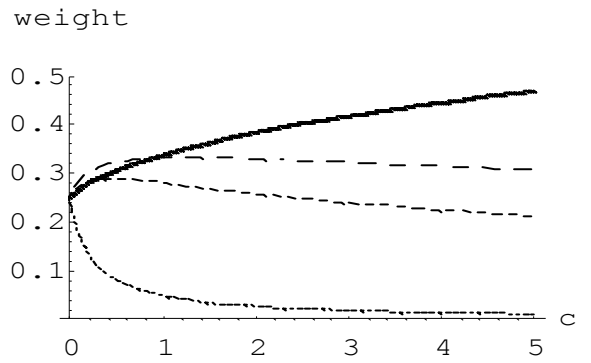

Figure 1.1: Change of the degree of importance derived from $A_{L}$

$$
A_{P}=\left(\begin{array}{cccc}
1 & 2.71 & 1.39 & 1 \\
.369 & 1 & .0975 & .0699 \\
.717 & 10.3 & 1 & 1 \\
1 & 14.3 & 1 & 1
\end{array}\right), r \mathrm{CI}_{P(4)}=0.126, m=m(1)
$$

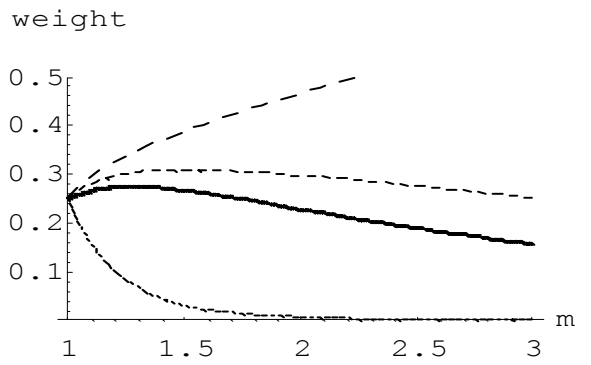

Figure 1.2: Change of the degree of importance derived from $A_{P}$

\subsection{Discriminating sensitivity}

One of the principal purposes of the AHP is to quantify the degree of importance for each alternative and

\footnotetext{
${ }^{4}$ As for the matrix whose size $h=5$ to 7 , we counted the sample whose rank reversed between the highest and the second highest weighted alternatives.
} 
to discriminate an important alternative from others. Let $\boldsymbol{v}^{\mathbf{1}}$ and $\boldsymbol{v}^{\mathbf{2}}$ respectively denote the largest and

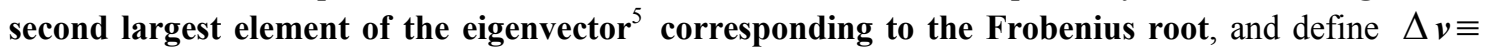
$v^{1}-v^{2}$ as the index of the discriminating sensitivity of scales $f_{L}$ and $f_{P}$. Furthermore, let $\Delta v_{\boldsymbol{L}}$ and $\Delta \boldsymbol{v}_{\boldsymbol{P}}$ denote the $\Delta v$ calculated by $f_{L}$ and $f_{P}$, respectively. Table 4 shows the result of comparisons between $\Delta v_{L}$ and $\Delta v_{P}$ for $h=4$. At $1 \%$ significant difference level in $X^{2}$-test, the number of samples holding $\Delta v_{L}<\Delta v_{P}$ was larger than that of $\Delta v_{L} \geq \Delta v_{P}$ for every matrix size, implying that $f_{L}$ was inferior to $f_{P}$ in discriminating sensitivity.

\begin{tabular}{|c|c|c|c|c|c|c|c|c|c|c|c|c|}
\hline mparisor & scrim! & tg sent & $\operatorname{cy}(4 x$ & & & & & & & & & Table \\
\hline \multirow[b]{3}{*}{ T-point } & \multicolumn{6}{|c|}{$f_{L}\left(x_{i j} ; 1\right)$} & \multicolumn{6}{|c|}{$f_{P}\left(x_{i j} ; m(1)\right)$} \\
\hline & \multicolumn{2}{|c|}{$v_{L}>v_{P}$} & \multicolumn{2}{|c|}{$v_{L}=v_{P}$} & \multicolumn{2}{|c|}{$v_{L}<v_{P}$} & \multicolumn{2}{|c|}{$v_{L}>v_{P}$} & \multicolumn{2}{|c|}{$v_{L}=v_{P}$} & \multicolumn{2}{|c|}{$v_{L}<v_{P}$} \\
\hline & Obs. & Ratio & Obs. & Ratio & Obs. & Ratio & Obs. & Ratio & Obs. & Ratio & Obs. & Ratio \\
\hline $1 \%$ & 99 & 44.4 & 2 & 0.900 & 122 & 54.7 & 83 & 31.3 & 1 & 0.380 & 181 & 68.3 \\
\hline $3 \%$ & 264 & 42.6 & 2 & 0.320 & 354 & 57.1 & 207 & 34.4 & 1 & 0.170 & 393 & 65.4 \\
\hline $5 \%$ & 406 & 40.3 & 2 & 0.200 & 599 & 59.5 & 369 & 36.0 & 2 & 0.200 & 653 & 63.8 \\
\hline $7 \%$ & 575 & 40.9 & 3 & 0.210 & 829 & 58.9 & 521 & 37.2 & 2 & 0.140 & 877 & 62.6 \\
\hline $10 \%$ & 813 & 40.6 & 3 & 0.150 & 1188 & 59.3 & 797 & 39.8 & 2 & 0.100 & 1206 & 60.2 \\
\hline $15 \%$ & 1237 & 41.2 & 3 & 0.100 & 1765 & 58.7 & 1207 & 40.2 & 3 & 0.100 & 1792 & 59.7 \\
\hline $20 \%$ & 1753 & 43.8 & 3 & 0.0700 & 2249 & 56.2 & 1656 & 41.1 & 3 & 0.0700 & 2367 & 58.8 \\
\hline $25 \%$ & 2216 & 44.3 & 4 & 0.0800 & 2787 & 55.7 & 2155 & 43.1 & 4 & 0.0800 & 2847 & 56.9 \\
\hline $100 \%$ & 7842 & 39.2 & 5 & 0.0300 & 12153 & 60.8 & 7842 & 39.2 & 5 & 0.0300 & 12153 & 60.8 \\
\hline
\end{tabular}

\section{Comparison between the linear and the power scales using the actual sample}

\subsection{CI}

In judging the consistency of a pair-wise comparison matrix $A$, Saaty insists that the allowable upper bound of $\mathrm{CI}$ is $10 \%$ of RI. In contrast, E.F. Lane and W.A.Verdini advocate that the allowable upper bound of $\mathrm{CI}$ is $1 \%, 5 \%$ and $10 \%$ of RI for the case $n=3,4$ and more than 5 , respectively, because the size of CI depends on matrix size $n$ (Lane and Verdini, 1989). However, these threshold values employing the certain ratio of RI as the allowable upper bound of $\mathrm{CI}$ have been criticized because their rationale is weak. Furthermore, since $r \mathrm{CI}_{L(h)}$ and $r \mathrm{CI}_{P(h)}$ have different distributions, comparing $f_{L}$ and $f_{P}$ with respect to the number of samples whose $\mathrm{CI}$ are smaller than the above-mentioned threshold value is not essential. Therefore, we evaluated $f_{L}$ and $f_{P}$ based on the cumulative number of samples with respect to each T-point of $r \mathrm{CI}_{L(4)}$ and $r \mathrm{CI}_{P(4)}$ as defined in Section 3.1.

Let $\boldsymbol{a} \mathbf{C I}_{\boldsymbol{L}}$ and $\boldsymbol{a} \mathbf{C I} I_{P}$ denote the $\mathbf{C I}$ obtained from the actual sample, $A_{L}$ and $A_{P}$, respectively. Table 5 summarizes each $r \mathrm{CI}_{L(4)}$ and $r \mathrm{CI}_{P(4)}$ corresponding to the T-point defined in Section 3.1, and the cumulative number of samples whose $a \mathrm{CI}_{L}$ and $a \mathrm{CI}_{P}$ are equal to or less than each $r \mathrm{CI}_{L(4)}$ and $r \mathrm{CI}_{P(4)}$. As can be seen in the table, $r \mathrm{CI}_{L(4)}>r \mathrm{CI}_{P(4)}$ from the $1 \%$ to $15 \%$-T-point; however, the cumulative number of samples generated by $f_{L}$ is smaller than that generated by $f_{P}$, at $1 \%$ significant difference level in $X^{2}$-test. This result showed that the CI of $A_{P}$ tended to cluster nearer to 0 than that of $A_{L}$ when the pair-wise comparison matrix $A_{L}$ and $A_{P}$ were generated by $f_{L}$ and $f_{P}$ from common $\left\{x_{i j}\right\}_{d}$, respectively. Implications that arise from these results are: $(i) f_{P}$ quantified individuals' feelings more consistently than $\operatorname{did} f_{L}$, and (ii) the number of samples with a large CI not being worthy of analysis may be reduced in applying the AHP to social research.

\subsection{Rank-reversal problem}

Table 6 shows (A, B), (A', B'), where A and A' are the cumulative number of samples generated by $f_{L}$ and $f_{P}$ respectively to each T-point among all (1409) actual samples, and among A and $\mathrm{A}^{\prime}$ samples in each category, B and B' are the cumulative number of samples where the rank reversal occurred with respect to the change of $c$ and $m(c)$ over the range shown in Equation (4). As can be seen, the number of rank-reversal samples was 57 among all $A_{L}$, and 43 among all $A_{P}$; this difference seemed to be small.

5 standardized 1 by $l_{1}$-norm 
Distribution of $a \mathrm{CI}_{L}$ and $\boldsymbol{a} \mathrm{CI}_{P}$

\begin{tabular}{|c|c|c|c|c|c|c|}
\hline \multirow[b]{2}{*}{ T-point } & \multicolumn{3}{|c|}{$f_{L}\left(x_{i j} ; 1\right)$} & \multicolumn{3}{|c|}{$f_{P}\left(x_{i j} ; m(1)\right)$} \\
\hline & $r \mathrm{CI}_{L(4)}$ & Obs. & Ratio & $r \mathrm{CI}_{P(4)}$ & Obs. & Ratio \\
\hline $1 \%$ & 0.04741 & 302 & $21.4 \%$ & 0.03277 & 379 & $26.9 \%$ \\
\hline $3 \%$ & 0.08662 & 430 & $30.5 \%$ & 0.06862 & 549 & $39.0 \%$ \\
\hline $5 \%$ & 0.1141 & 599 & $42.5 \%$ & 0.09807 & 717 & $50.9 \%$ \\
\hline $7 \%$ & 0.1448 & 657 & $46.6 \%$ & 0.1251 & 731 & $51.9 \%$ \\
\hline $10 \%$ & 0.1781 & 787 & $55.9 \%$ & 0.1648 & 932 & $66.2 \%$ \\
\hline $15 \%$ & 0.2318 & 949 & $67.4 \%$ & 0.2234 & 1016 & $72.1 \%$ \\
\hline $20 \%$ & 0.2865 & 1029 & $73.0 \%$ & 0.2899 & 1106 & $78.5 \%$ \\
\hline $25 \%$ & 0.3421 & 1102 & $78.2 \%$ & 0.3458 & 1195 & $84.8 \%$ \\
\hline $100 \%$ & -- & 1409 & $100.0 \%$ & -- & 1409 & $100.0 \%$ \\
\hline
\end{tabular}

Number of samples and their ratios: Rnak reversed

Table 6

\begin{tabular}{|c|c|c|c|c|c|c|}
\hline \multirow[b]{2}{*}{ T-point } & \multicolumn{3}{|c|}{$f_{L}\left(x_{i j} ; 1\right)$} & \multicolumn{3}{|c|}{$f_{P}\left(x_{i j} ; m(1)\right)$} \\
\hline & $\mathrm{A}$ & $\mathrm{B}$ & $\mathrm{B} / \mathrm{A}$ & $\mathrm{A}^{\prime}$ & $\mathrm{B}^{\prime}$ & $\mathrm{B}^{\prime} / \mathrm{A}^{\prime}$ \\
\hline $1 \%$ & 302 & 0 & $0 \%$ & 379 & 0 & $0 \%$ \\
\hline $3 \%$ & 430 & 1 & $0.233 \%$ & 549 & 0 & $0 \%$ \\
\hline $5 \%$ & 599 & 2 & $0.334 \%$ & 717 & 0 & $0 \%$ \\
\hline $7 \%$ & 657 & 3 & $0.457 \%$ & 731 & 0 & $0 \%$ \\
\hline $10 \%$ & 787 & 4 & $0.508 \%$ & 932 & 0 & $0 \%$ \\
\hline $15 \%$ & 949 & 4 & $0.422 \%$ & 1016 & 6 & $0.591 \%$ \\
\hline $20 \%$ & 1029 & 5 & $0.486 \%$ & 1106 & 16 & $1.45 \%$ \\
\hline $25 \%$ & 1102 & 9 & $0.817 \%$ & 1195 & 18 & $1.51 \%$ \\
\hline $100 \%$ & 1409 & 57 & $4.05 \%$ & 1409 & 43 & $3.05 \%$ \\
\hline
\end{tabular}

However, insofar as the samples whose $a \mathrm{CI}_{L}$ and $a \mathrm{CI}_{P}$ were smaller than the 5\%-T-point, which is nearly equal to 0.1 , no rank reversal occurred among $A_{P}$, while two occurred among $A_{L}$, as shown in Example 2. Furthermore, among $A_{L}$, rank reversal occurred even though such samples' CIs were small enough to be included in the group of "consistent" sample; in contrast, among $A_{P}$, rank reversal was never seen in the consistent group.

Figures 2.1, 2.2 shows rank-reversal examples whose CI is small and whose reversals occurred in the neighborhood of $c=1$. In these cases, $f_{L}$ is approximately equivalent to Saaty's 1-9 linear scale. Furthermore, in Example 2, the rank reversed between primal alternatives, rendering preference order derived by $f_{L}$ untrustworthy.

Example 2

$A_{L}=\left(\begin{array}{cccc}1 & 3 & 1 / 3 & 1 \\ 1 / 3 & 1 & 1 / 3 & 1 / 7 \\ 3 & 3 & 1 & 1 \\ 1 & 7 & 1 & 1\end{array}\right), a \mathrm{CI}_{L}=0.0819, c=1$

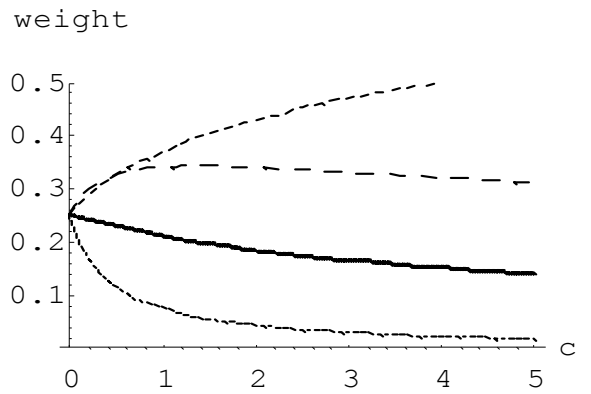

Figure 2.1: Change of the degree of importance derived from $A_{L}$

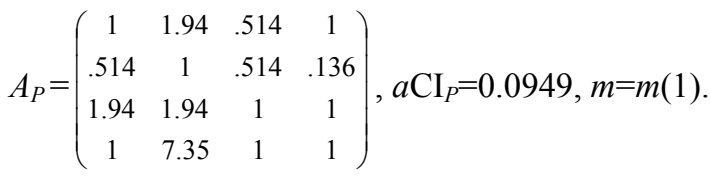

weight

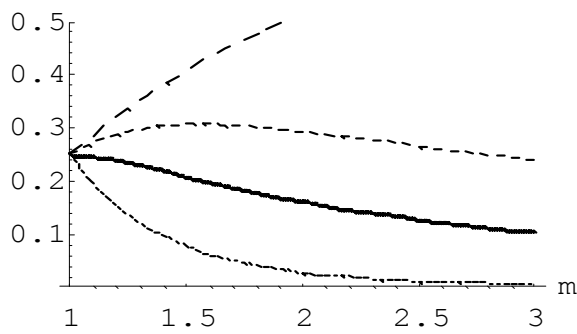

Figure 2.2: Change of the degree of importance derived from $A_{P}$

In addition to the robustness of $f_{P}$ in the consistent group, the abruptness in the increase in the number of rank-reversal samples in the group with CIs larger than the $15 \%$-T-point coincided with the number of 
rank-reversal samples. Leaving aside the discussion about the upper bound of acceptable CI, this coincidence might serve a good criterion for establishing the upper bound.

\subsection{Discriminating sensitivity}

Table 7 shows the results of comparisons between $\Delta v_{L}$ and $\Delta v_{P}$ for the 1409 actual samples in the same way as the analysis for the random sample in Section 3.3. As can be seen, both the 143 cases out of 182 samples of $f_{L}$ and the 143 cases out of 194 samples of $f_{P}$ are 1 matrix at the intersection of the 1\%-T-point row and the $\Delta \mathrm{v}_{L}=\Delta \mathrm{v}_{P}$ column. Additionally, the 235 cases out of the 247 samples of both $f_{L}$ and $f_{P}$ are $v^{1}=v^{2}$, resulting in $\Delta \mathrm{v}_{L}=\Delta \mathrm{v}_{P}=0$ at the intersection of the $100 \%$-T-point row and the $\Delta \mathrm{v}_{L}$ $=\Delta \mathrm{v}_{P}$ column. These results, contrary to those of the random sample shown in Table 4, imply that the actual sample obtained from the survey on public opinion includes responses whose pair-wise comparison data $\left\{x_{i j}\right\}_{d}$ are all 0 or responses with more than two "tie" alternatives weighted most. On the contrary, in the comparison of $f_{L}$ and $f_{P}$, the remaining samples, except for the responses whose pair-wise comparison data $\left\{x_{i j}\right\}_{d}$ are all 0 , show that the number of samples satisfying $\Delta \mathrm{v}_{L}<\Delta \mathrm{v}_{P}$ is larger than that satisfying $\Delta v_{L} \geq \Delta v_{P}$, at $1 \%$ significance level in $X^{2}$-test. Thus, $f_{L}$ is inferior to $f_{P}$ in discriminating sensitivity.

Comparison of discriminating sensitivity

Table 7

\begin{tabular}{|c|c|c|c|c|c|c|c|c|c|c|c|c|}
\hline \multirow[b]{3}{*}{ T-point } & \multicolumn{6}{|c|}{$f_{L}\left(x_{i j} ; 1\right)$} & \multicolumn{6}{|c|}{$f_{P}\left(x_{i j} ; m(1)\right)$} \\
\hline & \multicolumn{2}{|c|}{$v_{L}>v_{P}$} & \multicolumn{2}{|c|}{$v_{L}=v_{P}$} & \multicolumn{2}{|c|}{$v_{L}<v_{P}$} & \multicolumn{2}{|c|}{$v_{L}>v_{P}$} & \multicolumn{2}{|c|}{$v_{L}=v_{P}$} & \multicolumn{2}{|c|}{$v_{L}<v_{P}$} \\
\hline & Obs. & Ratio & Obs. & Ratio & Obs. & Ratio & Obs. & Ratio & Obs. & Ratio & Obs. & Ratio \\
\hline $1 \%$ & 46 & 15.2 & 182 & 60.3 & 74 & 24.5 & 47 & 12.4 & 194 & 51.2 & 138 & 36.4 \\
\hline $3 \%$ & 64 & 14.9 & 194 & 45.1 & 172 & 40.0 & 77 & 14.0 & 197 & 35.9 & 275 & 50.1 \\
\hline $5 \%$ & 87 & 14.5 & 211 & 35.2 & 301 & 50.3 & 99 & 13.8 & 212 & 29.57 & 406 & 56.6 \\
\hline $7 \%$ & 105 & 16.0 & 212 & 32.3 & 340 & 51.8 & 100 & 13.7 & 214 & 29.27 & 417 & 57.1 \\
\hline $10 \%$ & 146 & 18.6 & 218 & 27.70 & 423 & 53.8 & 160 & 17.2 & 221 & 23.71 & 551 & 59.1 \\
\hline $15 \%$ & 205 & 21.6 & 225 & 23.71 & 519 & 54.7 & 189 & 18.6 & 221 & 21.75 & 606 & 59.7 \\
\hline $20 \%$ & 231 & 22.5 & 226 & 21.96 & 572 & 55.6 & 244 & 22.1 & 229 & 20.71 & 633 & 57.2 \\
\hline $25 \%$ & 285 & 25.9 & 226 & 20.51 & 591 & 53.6 & 314 & 26.3 & 229 & 19.16 & 652 & 54.6 \\
\hline $100 \%$ & 469 & 33.3 & 247 & 17.530 & 693 & 49.2 & 469 & 33.3 & 247 & 17.530 & 693 & 49.2 \\
\hline
\end{tabular}

\section{Concluding remarks}

The AHP is a support system for decision making with subjective data as input and quantified data as output. To date, various judgment scales for a pair-wise comparison have been proposed and evaluated from the perspective of appropriateness for representing each DM's perception. In particular, Saaty's linear scale and Lootsma's power scale have often been compared and have generated much discussion. However, the weight obtained from a pair-wise comparison matrix cannot be verified theoretically, making evaluation of these two scales quite difficult. What is worse, this evaluation has not been able to be easily conducted because the CI of each scale, whose size is one of the criteria of evaluation, cannot simply be compared because of the difference of the properties of these two scales.

This study evaluated Saaty's linear scale and the power scale using two types of data, non-biased random sample and biased actual samples, from the point of view of appropriateness for representing each DM's feeling, where the criteria of appropriateness are the size of CI, robustness with respect to the change of the range of each scale, and discriminating sensitivity of each scale. In order to make comparisons between the two scales fair, the T-point was defined based on the relative size of the CI among all random samples rather than on its absolute value. The results of this study provide some evidence that, as a judgment scale, the power scale is preferable to Saaty's linear scale.

Nevertheless, several issues remain. ( $i$ ) How do we determine the base of a power scale? - Which base $m$ is the most appropriate for representing individuals' perceptions? (ii) How should we generate random samples?-On which interval I should they be based, and in accordance with which distribution should they follow? (iii) How would actual samples in a matrix other than $4 \times 4$ behave?

The first issue is which base to use for the power scale. In this study, we set $m=m(1)(\cong 1.3945)$; therefore, 
$f_{L}\left(x_{i j} ; 1\right)>f_{P}\left(x_{i j} ; m(1)\right)$ holds when $0 \leq x_{i j} \leq 6$ (corresponding to $1 \leq a_{i j}<7$ for Saaty's scale). This setting of $m$ is likely to make the distribution of the CI of $A_{P}$ clustered nearer to 0 than that of $A_{L}$. Additionally, the discriminating sensitivity of the power scale is superior to Saaty's linear scale. Furthermore, concerning the robustness with respect to the change of the range of each scale, the power scale was also superior to Saaty's linear scale. Thus, we might conclude that the power scale functioned better than Saaty's linear scale as the representation of each DM's perception. Whereas $m(1)$ seems to be a good option for defining the power scale in comparison with Saaty's linear scale, we cannot conclude that $m(1)$ is the best base for representing each DM's perception in the process of a pair-wise comparison. Determining the best base requires further analysis; other criteria besides $\mathrm{CI}$, rank reversal and discriminating sensitivity must be investigated.

The second issue is how to generate the random sample. In this study, the random sample was generated by selecting numbers from the set $\{-8, \ldots,-1,0,1, \ldots, 8\}$ in accordance with uniform randomness. In addition, we compared two scales through either $(i)$ a random sample generated on the basis of a set $\{-5$, $-3,-1,0,1,3,5\}$ in accordance with uniform randomness, or (ii) a random sample generated on the basis of a set $\{-8, \ldots,-1,0,1, \ldots, 8\}$ whose distribution follows $\mathrm{N}\left(0,4.2231^{6}\right)$. Leaving out the detail of the results, we note that, in either case, results similar to those of the analyses in Section 3 were obtained. However, both the random sample defined in Section 2.2.1 and the two aforementioned random samples satisfy weak single-peakedness and symmetry, possibly affecting the results. On the other hand, samples obtained from the application of the AHP to a realistic decision-making process do not always satisfy these two properties. Thus, further analysis using a random sample following a different distribution is necessary.

The third issue would be the size of actual sample. In this study, the matrix size of the actual sample was only $4 \times 4$; therefore, further investigations from at least $3 \times 3$ to $7 \times 7$ matrices are needed.

\footnotetext{
${ }^{6}$ standard deviation of the $\left\{x_{i j}\right\}_{d}$
} 


\section{References}

Aupetit, B. and Genest, C. (1993) "On Some Useful Properties of the Perron Eigenvalue of a Positive Reciprocal Matrix in the Context of the Analytic Hierarchy Process," European Journal of Operational Research, 70, 263-268.

Crawford, G. and Williams, C. (1985) “A Note on the Analysis of Subjective Judgment Matrices," Journal of Mathematical Psychology, 29, 387-405.

Harker, P. T. and Vargas, L. G. (1987) “The Theory of Ratio Scale Estimation: Saaty's Analytic Hierarchy Process," Management Science, 33, 1383-1403.

Van Den Honert, R. C. and Lootsma, F. A. (1997) "Group Preference Aggregation in the Multiplicative AHP, The Model of the Group Decision Process and Pareto Optimality," European Journal of Operational Research, 96, 363-370.

Kumar, N. V. and Ganesh, L. S. (1996) "A Simulation-based Evaluation of the Approximate and the Exact Eigenvector Methods Employed in AHP,” European Journal of Operational Research, 95, 656-662.

Lane, E. F. and Verdini, W. A. (1989) “A Consistency Test for AHP Decision Makers,” Decision Science, 20, 575-590.

Lootsma, F. A. (1989) “Conflict Resolution via Pairwise Comparison of Concessions,” European Journal of Operational Research, 40, 109-116.

Lootsma, F. A. (1991) "Scale Sensitivity and Rank Preservation in a Multiplicative Variant of the Analytic Hierarchy Process," Delft University of Technology, Report of the Faculty of Technical Mathematics, 91-20.

Saaty, T. L. (1977) “A Scaling Method for Priorities in Hierarchical Structures,” Journal of Mathematical Psychology, 15, 234-281.

Saaty, T. L. and Bennett, J. P. (1977) “A Theory of Analytical Hierarchies Applied to Political Candidacy," Behavioral Science, 22, 237-245.

Saaty, T. L. (1983) "Priority Setting in Complex Problems," IEEE Transactions on Engineering Management, EM-30, 140-155.

Saaty, T. L. (1980) The Analytic Hierarchy Process, McGraw-Hill.

Schenkerman, S. (1994) “Avoiding Rank Reversal in AHP Decision-support Models,” European Journal of Operational Research, 74, 407-419.

Vargas, L. G. (1994) "Reply to Schenkerman's Avoiding Rank Reversal in AHP Decision Support Models," European Journal of Operational Research, 74, 420-425. 